\title{
Real-vehicle integration of driver support application with IPv6 GeoNetworking
}

\author{
Satoru Noguchi*†, Manabu Tsukada*, Ines Ben Jemaa*, Thierry Ernst* \\ ${ }^{*}$ INRIA Rocquencourt, IMARA project-team, France \\ Email: \{satoru.noguchi, manabu.tsukada, ines.benjemaa, thierry.ernst\} @inria.fr \\ ${ }^{\dagger}$ Graduate school of Information Science, Nara Institute of Science and Technology, Japan \\ Email: satoru-n@ is-naist.jp
}

\begin{abstract}
One of the essential usage of Intelligent Transportation Systems (ITS) applications is to provide road traffic information to vehicle drivers for road safety and efficient drive. For this usage, it is necessary to integrate geographical routing mechanisms in vehicular ad hoc network (VANET) into ITS applications. In this paper, we design and implement an ITS application which relies on IPv6 GeoNetworking; a geographical addressing and routing mechanism developed in the GeoNet project. Our application supports realistic use case scenarios, therefore we integrated it into INRIA's vehicular platform. The system has publicly been demonstrated in realistic scenarios.
\end{abstract}

\section{INTRODUCTION}

Intelligent Transportation Systems (ITS) applications aim to provide road users with improved traffic safety, traffic efficiency, and additional value. They run on Application Units (AUs) connected to On-Board Units (OBUs) in vehicles, Roadside Units (RSUs) in roadside infrastructure, and Internet hosts. IPv6 is considered as a mandatory communication protocol in ITS network because of its capabilities of mobility support and huge address space [1]. One of the essential usage of ITS applications is to provide road traffic information with vehicle drivers for safety and efficient drive possibly through in-vehicle Human Machine Interfaces (HMI).

ITS applications are significantly different from regular applications; they need to deliver messages to group of vehicles within a particular area specified with geographical position because most road traffic information is related to the geographical position of the vehicle. For example, if a vehicle detects a road traffic event (e.g. accident, congestion, etc), it reports the event to an ITS centre and the centre assigns a RSU, which locates in relevant area, to inform approaching vehicles of the event. IPv6 GeoNetworking developed in the GeoNet project, an European project started from 2008 and finished in $2010,{ }^{1}$ provides such geographical routing mechanism. It combines IPv6 and Vehicular Ad hoc NETworks (VANETs) and routes packets using geographic position via wireless multihop communication [2].

In order to address benefits, requirements, and issues of exploiting the geographical routing for ITS, we design and implement an ITS application exploiting IPv6 GeoNetworking capabilities. Thanks to its geographical routing functionality, our application effectively delivers location-specific messages

\footnotetext{
${ }^{1}$ The FP7-funded European project GeoNet http://www.geonet-project.eu/
}

to a certain area instead of regular broadcasting in VANET. We integrate the proposing system into our real vehicle testbed.

The remainder of this paper is organized as follows. Section II overviews the ITS network, existing efforts of developing ITS applications, and IPv6 GeoNetworking completed in the GeoNet project. Section III describes use cases and functional requirements of our application. Section IV is dedicated to our propose system architecture for our application harmonized with IPv6 GeoNetworking. We then present integration and demonstration results in our real vehicle testbed in Section VI. After discussing issues in Section VII, Section VIII finally concludes the paper.

\section{RELATED WORK}

The ITS network combines VANETs and the Internet. Fig. 1 shows the simplified network topology composed of sets of nodes: OBU, RSU, and AU. Several ITS projects and standards organizations have been developing these nodes (ITS station) conforming to ISO/ETSI's ITS station reference architecture. OBU, which is a device installed in vehicles, and RSU, a device installed along the roadside, behave as mobile routers and access routers that may compose VANETs, whereas AUs run ITS applications. The RSU allows OBUs to access to the Internet. IPv6 works as the network layer communication protocol. Based on the ITS network, a wide variety of ITS applications has been defined in ITS projects and standards organizations. They have been categorized into three types of applications: road safety, traffic efficiency, and others (infotainment or value-added services) [3], [4], [5].

The GeoNet project has developed a reference specification of IPv6 operating over GeoNetworking and conforming C2CNet specification [2], [6]. C2CNet, specified by the CAR 2 CAR Communication Consortium ${ }^{2}$, is a communication layer dedicated to car-to-car communication and is located between IPv6 and the link layer. It supports geographical addressing and routing by means of encapsulating IPv6 packet with a new $\mathrm{C} 2 \mathrm{CNet}$ header, which contains source and destination C2CNet identifier, source and destination geographic position. Although $\mathrm{C} 2 \mathrm{CNet}$ layer itself exchanges packets with non-IP messaging, the GeoNet project has defined how to transmit IPv6 packet over C2CNet ("IPv6 over C2CNet").

\footnotetext{
${ }^{2}$ CAR 2 CAR Communication Consortium. http://www.car-to-car.org/
} 


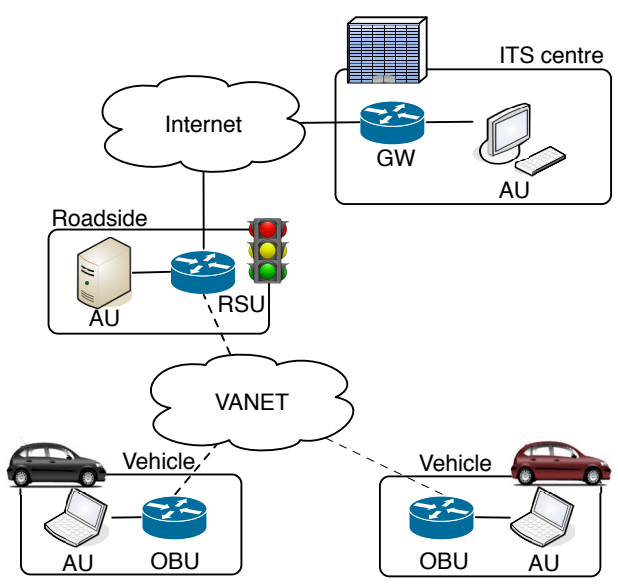

Fig. 1. ITS network

This is performed transparently to the upper layers; In IPv6 GeoNetworking-enabled VANETs, each node is assigned a $\mathrm{C} 2 \mathrm{CNet}$ identifier. When an originator node constructs an IPv6 packet with destination node's IPv6 address, the C2CNet layer encapsulates the packet with $\mathrm{C} 2 \mathrm{C}$ header with the IP next hop's $\mathrm{C} 2 \mathrm{CNet}$ identifier. The $\mathrm{C} 2 \mathrm{CNet}$ layer thereby makes the routing decision with the C2CNet identifier and nodes' geographic location. IPv6 is thus used in a regular manner; when upper layers issue IPv6 unicast, multicast, and anycast packets, the IPv6 over $\mathrm{C} 2 \mathrm{CNet}$ mechanism delivers them with GeoUnicast, GeoBroadcast/TopoBroadCast, and GeoAnycast, respectively [2].

\section{DRIVER AWARENESS APPLICATION ENHANCED WITH IPv6 GEONETWORKING}

In this section, we define two essential use case scenarios for supporting drivers and discuss the functional requirements of ITS applications for such use cases. They can be categorized into road safety application and traffic efficiency application. The first one is Road traffic event detection and dissemination; when a vehicle detects a particular road traffic event (e.g. accident, approaching emergency vehicle, etc), it reports the description of the event to an ITS centre. The ITS centre shows the occurrence of the event to ITS operators and inform relevant vehicles of the event. The second scenario is Road traffic Information dissemination from ITS centre; an ITS operator in an ITS centre informs about a particular road traffic event (e.g. road congestion) to relevant vehicles, which reside within the relevant area of the event. Both scenarios therefore rely on three types of vehicular communications; Vehicle-Vehicle, Vehicle-Roadside, and Vehicle-Internet communication defined in [2].

In each scenario, dissemination messages are delivered to vehicles within the relevant geographical area (GeoDestination). It covers an appropriate range of roads through which the receivers approach the point of event. The size of the area is configured depending on the type of event, receivers' possible

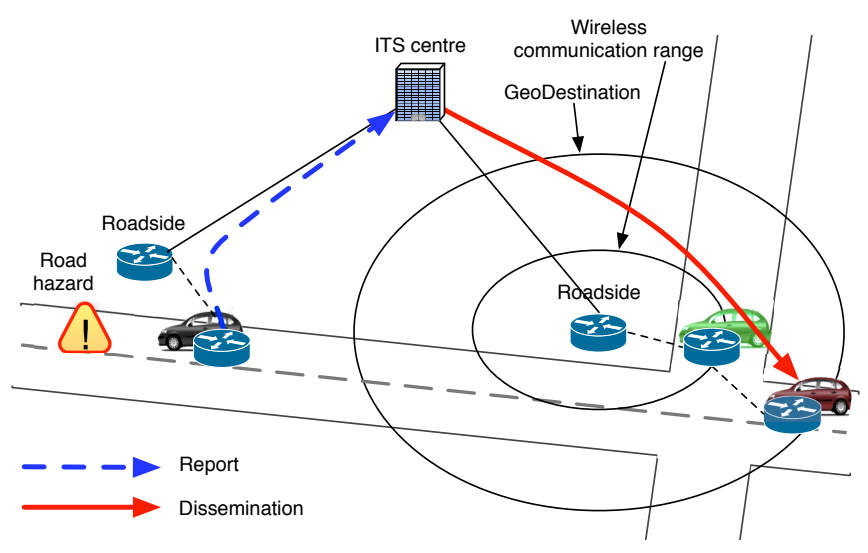

Fig. 2. Road traffic event detection and dissemination scenario

driving speed, and the characteristics of possible destination area. For example, in urban area composed of narrow streets, it is assumed that the driving speed is relatively slow and it may regularly be congested. In such a situation the GeoDestination can be small for saving bandwidth, and vice versa.

Fig. 2 overviews the Road traffic event detection and dissemination scenario. Regarding the Road traffic Information dissemination scenario, it only uses the dissemination mechanism in this Figure.

The functional requirements of ITS applications are defined according to these scenarios. Three types of functions realize the application; (i) detecting and reporting road traffic events (Reporter), (ii) aggregating and disseminating the road traffic event to relevant vehicles (Disseminator), and (iii) receiving messages from the Disseminator and notifying drivers of the event (Receiver). The receiver and reporter shall be integrated into vehicles. On the other hand, the disseminator shall be equipped with AUs in ITS centre.

From the point of view of communication, AUs hosting the ITS application shall be reachable from one another. It means that roadside infrastructures shall provide Internet access to vehicles. The reporter shall know the communication endpoints of an appropriate disseminator (i.e. IPv6 address and port). As vehicles are sets of mobile nodes, the reporter shall be statically configured with a set of appropriate disseminators. Otherwise, it shall have some mechanism to dynamically find an appropriate disseminator.

\section{System ARChiteCTURE}

Our application consists of two types of software entities located in AUs: clients in vehicle and a server in an ITS centre. The client acts as the reporter and receiver at the same time, whereas the server acts as the disseminator. To deliver messages to a particular geographical area, applications transparently exploit IPv6 GeoNetworking developed in the GeoNet project. Thanks to the geographical routing mechanism, messages are delivered to GeoDestination independently of routers' wireless communication range. We particularly exploit the GeoBroadcast mechanism that enables to send 


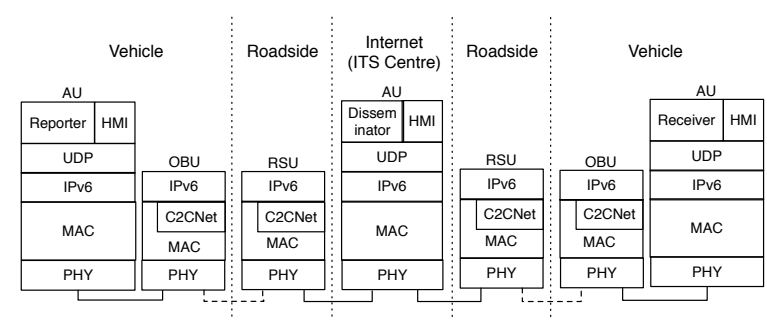

Fig. 3. Protocol Architecture

packets from a node to all nearby nodes located within a particular geographical area [6].

While OBUs and RSUs have IPv6 GeoNetworking functionality, the application itself is integrated into AUs, which only have a regular IPv6 stack. Fig. 3 shows the overall protocol architecture of our system. Configurations of each station are described as follows:

Vehicle: Vehicles are equipped with an OBU that has IPv6 GeoNetworking and an AU that contains regular IPv6 stack. For station-internal communication between $\mathrm{AU}$ and $\mathrm{OBU}$, regular Ethernet is used. WLAN is used for station-external communication between OBU and other OBUs/RSUs. The client application consists of two main components and one support component: reporter, receiver, and HMI, respectively. The reporter automatically detects road traffic events and reports them to the disseminator via UDP unicast. The receiver receives messages from the disseminator via IPv6 multicast, which are encapsulated to GeoBroadcast packets, and then notify vehicle drivers. Thus AUs join an IPv6 multicast group. Both main components inform drivers of road traffic event detection and message reception though the GUI windows provided by the HMI.

Roadside: The roadside only has a RSU that supports both IPv6 GeoNetworking and regular IPv6 routing. The RSU encapsulates regular IPv6 multicast packets delivered from the Internet and GeoBroadcasts to OBUs via its wireless network interface. Even though a RSU does not cover the necessary range for delivering GeoBroadcast packets, the packets is forwarded by nearby vehicles thanks to the multihop communication mechanism in IPv6 over C2CNet.

Centre: ITS centre contains an AU connected to the Internet. In the $\mathrm{AU}$, the server application has one main component and one support component: disseminator and HMI, respectively. The disseminator aggregates road traffic event reported by clients via regular UDP unicast, and propagates them to other receiver clients via IPv6 multicast. The disseminator also informs ITS operators of road traffic event detection though the GUI window of the HMI.

\section{A. Communication sequence}

Messaging sequences shown in Fig. 4 are as follows:

1) RSUs and the disseminator establish an IPv6 multicast tunnel. RSUs are configured for forwarding IPv6 multicast from the Internet to VANET.

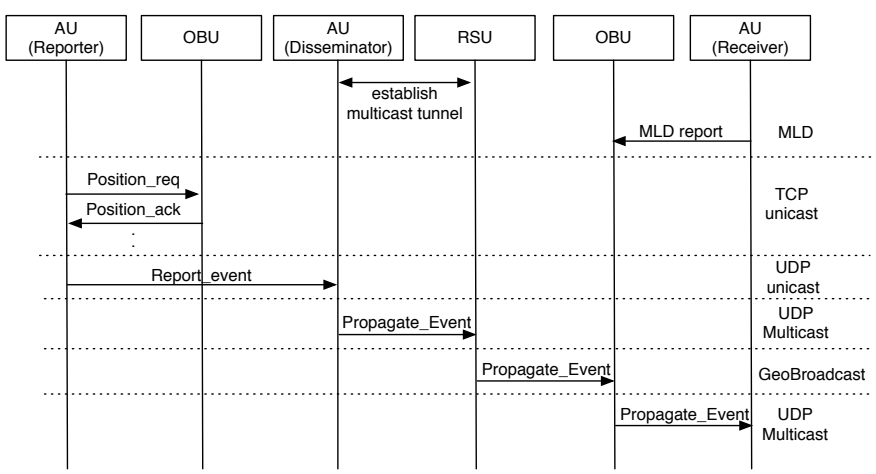

Fig. 4. Messaging sequence. Report_event is used only in the Road traffic event detection and dissemination scenario.

2) Reporter periodically gets the vehicle's current geographical position from OBU (Position_req, Position_ack) via TCP unicast.

3) Receiver join a particular global scope IPv6 multicast group by issuing MLDv2 Multicast Lister Report to OBU.

4) In the case of Road traffic event detection and dissemination, when a reporter detects a road traffic event, it reports descriptions of the event to the disseminator with UDP unicast (Report_event) via the reachable RSU.

5) The disseminator receives the report and displays the position and descriptions of the event to ITS operators through HMI.

6) The disseminator periodically sends IPv6 multicast packets to RSUs through the multicast tunnel (Propagate_Event) for a certain period of time.

7) RSUs receive the IPv6 multicast packets and forwards them via $\mathrm{C} 2 \mathrm{CNet}$ layer, which generates GeoBroadcast packets. The size of GeoDestination is determined from the IPv6 multicast group address.

8) OBU's C2CNet layer receives GeoBroadcast packets and decapsulate them. Regular IPv6 multicast packets are stripped out of the C2CNet header. It passes the IPv6 packets to its IPv6 stack. The IPv6 stack finally sends the packes to receiver through regular Ethernet.

9) Receiver notifies its driver of the reception of messages.

\section{IMPLEMENTATION}

We implemented the proposed system and integrated it into our real vehicle testbed in INRIA rocquencourt that includes three vehicles equipped with one OBU and one AU, two RSUs, and another AU as ITS centre. The system is therefore capable of testing multi-hop communication in VANET. OBUs and RSUs are Alix $3 \mathrm{~d} 3$ embedded PCs equipped with an Ethernet port as an ingress interface which is connected to station-local wired network, and a mini-pci wireless card (Atheros AR5414 $802.11 \mathrm{a} / \mathrm{b} / \mathrm{g} \operatorname{Rev} 01)$ used as an egress interface. Ubuntu 9.0.4 (kernel 2.6.29.6) is installed to both of them. They also have capabilities to get their current geographical position; OBUs are connected to in-vehicle GPS receiver (Trimble 
AgGPS 323), with which gpsd ${ }^{3}$ in OBU provides vehicles' current location, and RSUs are statically configured with their position. AUs are conventional PCs.

OBUs and RSUs have an IPv6 protocol stack including a multicast daemon and a $\mathrm{C} 2 \mathrm{CNet}$ layer. The multicast daemon enables multicast forwarding and records the multicast groups on their ingress interfaces. The IPv6 multicast tunnel between RSUs and the ITS centre is statically configured for now. Regarding AUs, although our application is able to run on any operating system as long as they support IPv6, MLDv2, and Java, we used Ubuntu 9.0.4 for operational reasons. Each vehicle $\mathrm{AU}$ is connected to its OBU through wired Ethernet, on the other hand, an AU in the ITS centre is directly connected the Internet.

The IPv6 GeoNetworking mechanism on OBUs and RSUs is implemented with TUN virtual interface that communicates with IPv6 and C2CNet. OBUs (RSUs) at first receive regular IPv6 packets on their ingress (egress) interface and pass the packets to $\mathrm{C} 2 \mathrm{CNet}$ module via the virtual interface. Then subsequent communication is performed in $\mathrm{C} 2 \mathrm{CNet}$ layer. The $\mathrm{C} 2 \mathrm{CNet}$ layer determines the type of communication (i.e. GeoUnicast or GeoBroadcast) from the first 8 bits of the destination IPv6 address. Regarding the GeoBroadcast generated from IPv6 multicast, the destination area is mapped to a particular IPv6 multicast address, such as "ff02::1" to a radius of $500 \mathrm{~m}$. The $\mathrm{C} 2 \mathrm{CNet}$ layer in intermediate OBUs and RSUs forward the packets with multi-hop manner. Destination OBUs (RSUs) receive the packets via their egress (ingress) interface and passes them to their ingress (egress) interface via the virtual interface as regular IPv6 packets.

The client application is implemented under JavaSE6 and JavaFX1.2, as shown in Fig. 5: the reporter and receiver components run on JavaSE whereas the HMI component runs on JavaFX. The client HMI displays the vehicle's name, current geographical position, detected road traffic events (reporter's function), and reported messages (receiver's function). As the road traffic event detection mechanism is out of focus of this paper, currently the event detection is statically performed in the application's configuration file by specifying the geographical position where the event is supposed to take place.

The server application is implemented under JavaSE6, JavaFX1.2 and Google Earth; the disseminator is developed under JavaSE and the HMI is composed of JavaFX and Google Earth. The server HMI has a local http server that overlays reported road traffic information on Google Earth by means of generating KML sentences, shown in Fig. 6.

\section{Field Demonstration}

We conducted a field demonstration of our system with the scenarios described in Section III. The main goal of this demonstration is to show effectiveness of integrating the ITS application on top of IPv6 GeoNetworking thanks to the following three features: GeoBroadcast generated from IPv6 multicast, Internet-to-Geographical area communication, and

\footnotetext{
${ }^{3}$ gpsd - a GPS service daemon. http://gpsd.berlios.de/
}
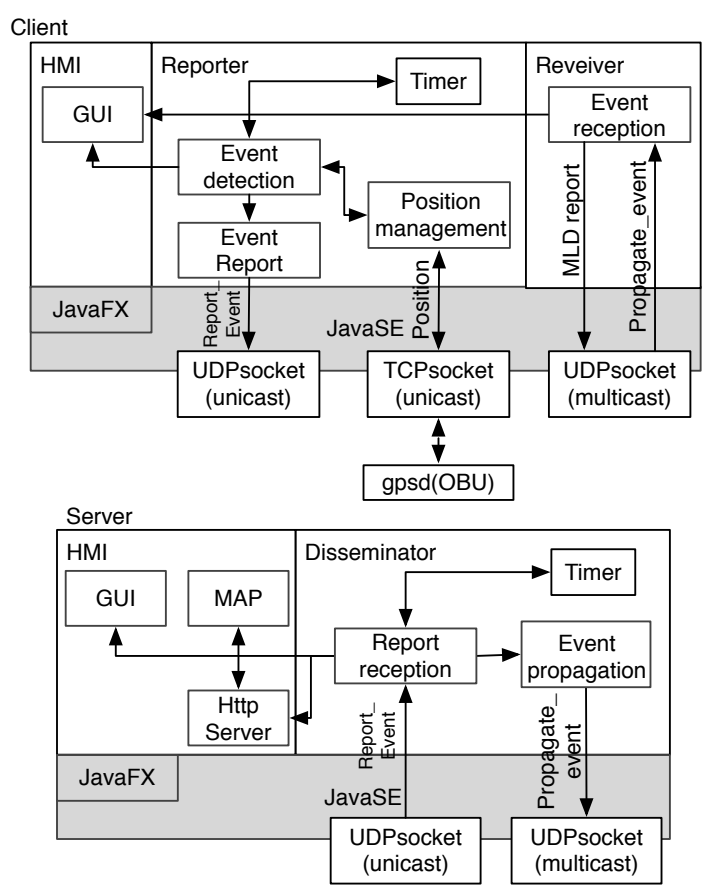

Fig. 5. Components' structure

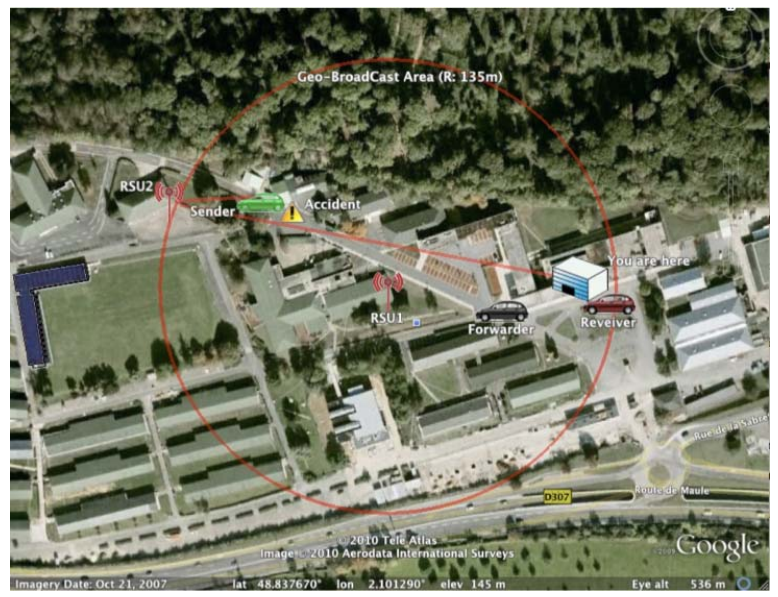

Fig. 6. Map view in ITS centre

multi-hop geographical routing at the $\mathrm{C} 2 \mathrm{CNet}$ layer. Following the scenario depicted in Fig. 2, one of the three vehicles (the forwarder) acts as a stationary vehicle at the boundary of wireless communication range of RSU so that it forwards GeoBroadcast packets to GeoDestination. Other two vehicles, which act as the reporter or receiver, go around a pre-specified route. When the reporter vehicle is close to the statically configured road hazard point, it reports the description of the event to the server running on an AU in the ITS centre via nearest RSU (RSU2). After receiving the report, the server in the centre periodically sends the event notification messages (Propagate_event) with IPv6 multicast to a relevant RSU (RSU1). RSU1 propagates the event to a GeoDestination with GeoBroadcast generated from the IPv6 multicast packets. The 
TABLE I

SYSTEM CONFIGURATION

\begin{tabular}{|c|c|c|}
\hline Entity & Parameter & Configuration \\
\hline \hline All & Multicast group address & FF0E::2 \\
\hline AU (Server) & Event propagation frequency & $10 \mathrm{~Hz}$ \\
\hline RSU, OBU & GeoBroadcast radius & $150 \mathrm{~m}$ \\
\hline AU (Client) & GPS position update interval & $1 \mathrm{sec}$ \\
\hline AU & HMI refresh interval & $1 \mathrm{sec}$ \\
\hline Vehicle & Driving speed & $<40 \mathrm{~km} / \mathrm{h}$ \\
\hline
\end{tabular}

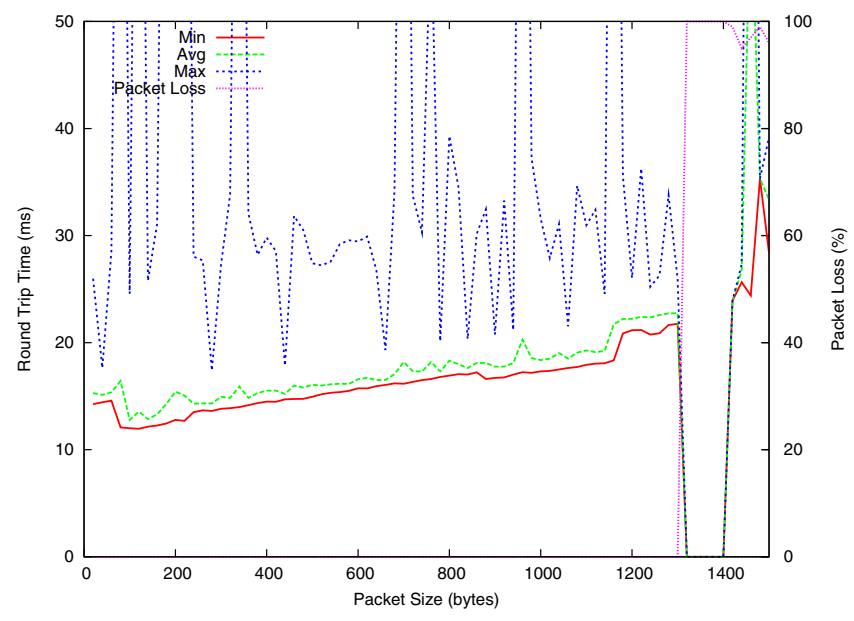

Fig. 7. Latency between RSU and OBU (multi-hop)

receiver vehicle receives the packets as long as it resides within the destination area. Regarding the other scenario, Road traffic information dissemination, the server can also send the traffic information messages (Propagate_info) at any time without report from reporters.

The system configuration is shown in Table I. Through several preliminary tests, we determined Event propagation frequency, which represents the frequency of the transmission of event propagation messages (Propagate_event) to $10 \mathrm{~Hz}$ in order to notify drivers of road traffic events as fast as possible via the clients' HMI. The range of GeoBroadcast is configured to a radius of $150 \mathrm{~m}$ of the location of RSU. One of our tests showed the average Round Trip Time (RTT) between RSU and OBU using IPv6 GeoNetworking on multi-hop was around $15 \mathrm{~ms}$, shown in Fig. 7. It means that even though the GeoBroadcast packets are propagated with multi-hop, it is delivered within $7.5 \mathrm{~ms}$ from RSU to OBU.

Our system has publicly been demonstrated at the final workshop of the GeoNet project at INRIA premises (January 2010, Rocquencourt, France) [7].

\section{DISCUSSION}

In this section, we discuss the design issues of ITS applications enhanced with IPv6 GeoNetworking. For large-scale ITS network containing several ITS centres and RSUs, messages sent from reporters should be delivered to an appropriate ITS centre which supports a particular service, organization, or geographical area. According to stringent latency requirements for ITS applications (e.g. from $10 \mathrm{~ms}$ to $1000 \mathrm{~ms}$ for road safety [8]), locating such a ITS centre must be done within a few milliseconds or before sending the report. Service Discovery technology, a mechanism to automatically discover communication endpoint from services' name and attributes, may be helpful for this issue [9].

ITS centres need to have ability to select appropriate RSUs which disseminate IPv6 multicast packets using GeoBroadcast. One proposal is to send IPv6 multicast to all RSUs. However, it might consume bandwidth in the case of large scale network.

The ITS station reference architecture, as mentioned in Section II, contains multiple access technologies and NEtwork MObilty support protocol (NEMO) [10]. This architecture comprises the IPv6 GeoNetworking mechanism and is assumed as a foundation of ITS, thus ITS applications must cooperate with the architecture.

\section{CONCLUSION}

In this paper, we designed an ITS application enhanced with IPv6 GeoNetworking developed in the GeoNet project. At first we described the necessity of IPv6 GeoNetworking for ITS applications. After showing typical use cases and requirements of ITS applications based on geographical routing, we designed and implemented our ITS application that consists of two entities; a server in an ITS centre and clients in vehicles. Our system was integrated into outdoor field test environment and successfully worked in realistic scenarios as the final demonstration of the GeoNet project at INRIA.

\section{ACKNOWLEDGMENT}

The work reported in this paper has been financed by the European Commission under the GeoNet project (grant agreement 216269). The authors would like to thank all the members of the GeoNet project.

\section{REFERENCES}

[1] T. Ernst, "The information technology era of the vehicular industry," SIGCOMM Comput. Commun. Rev., vol. 36, no. 2, pp. 49-52, 2006.

[2] GeoNet Project, D1.2 Final GeoNet Architecture Design, June 2010.

[3] CAR 2 CAR Communication Consortium, "CAR 2 CAR communication consortium manifesto, overview of the C2C-CC system, version 1.1," August 2007.

[4] ETSI, ETSI TR 102638 Technical Report, V1.1: Intelligent Transport Systems (ITS), Vehicular Communications (VC), Basic Set of Applications, Definitions, June 2009.

[5] CVIS project, D.CVIS.3.3 Architecture and System Specifications, http://www.cvisproject.org/, July 2007.

[6] M. Tsukada, I. B. Jemaa, H. Menouar, W. Zhang, M. Goleva, and T. Ernst, "Experimental evaluation for IPv6 over VANET geographic routing," in IWCMC '10: Proceedings of the 6th International Wireless Communications and Mobile Computing Conference, 2010, pp. 736741.

[7] GeoNet Project, D7.1 GeoNet Experimentation Results, June 2010.

[8] G. Karagiannis, R. Wakikawa, J. Kenney, C. J. Bernardos, and F. Kargl, "Traffic safety applications requirements," Internet Engineering Task Force, Internet-Draft draft-karagiannis-traffic-safety-requirements02.txt, February 2010, (Work in progress).

[9] A. N. Mian, R. Baldoni, and R. Beraldi, "A survey of service discovery protocols in multihop mobile ad hoc networks," Pervasive Computing, IEEE, vol. 8, no. 1, pp. $66-74$, Jan.-March 2009.

[10] V. Devarapalli, R. Wakikawa, A. Petrescu, and P. Thubert, "Network Mobility (NEMO) Basic Support Protocol," RFC 3963 (Proposed Standard), Internet Engineering Task Force, Jan. 2005. [Online]. Available: http://www.ietf.org/rfc/rfc3963.txt 